Background Because of increased transmission potential, the US Centres for Disease Control and Prevention (CDC) recommends prioritising high HIV viral load ( $\geq 50,000$ copies/mL, HVL) individuals for routine follow-up services, including linkage-to-care and partner services. However, little guidance exists on operationalizing this recommendation. In June 2014, the Baltimore City Health Department developed and implemented a pilot program to prioritise HVL individuals for follow-up services. The objective of this analysis is to describe the pilot program and evaluate process outcomes for follow-up services pre- and post-pilot program implementation.

Methods This pilot program was modelled after a protocol for responding to congenital syphilis. Disease Intervention Specialists (DIS) were trained to locate, administer partner services interviews and link-to-care HVL individuals with increased urgency and effort compared to other HIV cases. A DIS supervisor reviewed each HVL case before closing to ensure adequate response and documentation. We used a pre-post design to evaluate the pilot and compared linkage-to-care and contact tracing outcomes for HVL individuals post pilot implementation (postpilot, June 2014-January 2015) to a similar time period prior to implementation (pre-pilot, June 2013-January 2014).

Results There were 23 pre-pilot and 17 post-pilot HVL cases (n $=40$ ). DIS were more likely to link HVL individuals to care (59\% pre-pilot vs. 65\% post-pilot), and complete partner services interviews (39\% pre-pilot vs. 59\% post-pilot). Among HVL individuals who completed interviews, DIS were more likely to obtain sex partner meeting place information (33\% pre-pilot vs. $40 \%$ post-pilot) and locating information for disclosed sex partners (16\% pre-pilot vs. $39 \%$ post-pilot) in the post-pilot period compared to the pre-pilot period.

Conclusion This pilot program demonstrates one successful method to operationalize CDC guidelines regarding prioritisation of HVL individuals. Future work will evaluate additional outcomes of this program, including HIV testing among sex partners and at sex partner meeting places disclosed by HVL individuals.

Disclosure of interest statement The authors have no conflicts of interest to disclose.

\section{P17.24 DELAYED LINKAGE TO CARE IN A THIRD OF HIV POSITIVE INDIVIDUALS IN THE NETHERLANDS: OPPORTUNITIES TO IMPROVE THE SECOND STEP IN THE CASCADE OF CARE}

\begin{abstract}
${ }^{1,2}$ MG Van Veen, ${ }^{1} \mathrm{SCM}$ Trienekens, ${ }^{2} \mathrm{~T}$ Heijman, ${ }^{3} \mathrm{HM}$ Gotz, ${ }^{4} \mathrm{~S}$ Zaheri, ${ }^{1,5} \mathrm{G}$ Ladbury, ${ }^{6}$ D De Wit, ${ }^{2} \mathrm{JSA}$ Fennema, ${ }^{4} \mathrm{~F}$ De Wolf, ${ }^{1,7} \mathrm{MAB}$ Van Der Sande*. ${ }^{7}$ National Institute for Public Health and the Environment (RIVM), Centre for Infectious Disease Control, Bilthoven, The Netherlands; ${ }^{2}$ Public Health Service, Amsterdam, The Netherlands; ${ }^{3}$ Municipal Public Health Service Rotterdam-Rijnmond, The Netherlands; ${ }^{4}$ HIV Monitoring Foundation (SHM), Amsterdam, The Netherlands; ${ }^{5}$ EPIET, ECDC, Stockholm, Sweden; ${ }^{6}$ University of New South Wales, Centre for Social Research in Health; 'Julius Centre, University of Utrecht, The Netherlands
\end{abstract}

\subsection{6/sextrans-2015-052270.602}

Introduction The HIV cascade of care can identify missed opportunities to optimise control. The first step entails early testing, the second step is to ensure prompt linkage to care once diagnosed. To determine time to linkage to HIV-care following diagnosis at an STI centre, and to identify risk factors for delayed linkage.

Methods Patients newly diagnosed with HIV at STI clinics in the Netherlands were followed until linkage to care. Data were collected at time of diagnosis and at first consultation in care, including demographics, behavioural information, $\mathrm{CD}^{+}$counts and HIV viral load measurements. Delayed linkage to care was defined as $>4$ weeks between HIV diagnosis and first consultation.

Results 310 participants were included; the majority (90\%) men who have sex with men. For 259 participants (84\%) a date of first consultation in care was known; median time to linkage was 9 days (range 0-435). Overall, 95 (31\%) of participants were not linked within 4 weeks of diagnosis; among them, 44 were linked late and 51 were not linked at all by the end of study follow-up. Being young $(<25$ yrs $)$, having a non-Western ethnicity or lacking health insurance were independently associated with delayed linkage to care. Also, those being referred to care indirectly were more likely to have delayed linkage. Baseline $\mathrm{CD}^{+}{ }^{+}$count, viral load, perceived social support and stigma at diagnosis were not associated with delayed linkage. Risk behaviour and $\mathrm{CD}^{+}$counts declined between diagnosis and linkage to care.

Conclusions Although most newly diagnosed HIV patients were linked to care within 4 weeks, delay was observed for a third, with over half of them not yet linked at the end of follow-up. Vulnerable subpopulations (young, uninsured, ethnic minority) were at risk for delayed linkage. Testing those at risk is not sufficient, timely linkage to care needs to be assured.

Disclosure of interest statement This study was funded by the Netherlands Organisation for Health Research and Development (ZonMW) and the Ministry of Health, Welfare and Sport, the Netherlands. No pharmaceutical grants were received in the development of this study.

\section{P17.25 IMPACT ON COMPLIANCE WITH THE CHANGE OF FIRST LINE ANTI-RETROVIRAL DRUG REGIMENS AMONG PATIENTS ATTENDING ANTERETROVIRAL THERAPY CLINICS IN BLANTYRE, MALAWI}

Y Gadama*, S Sheikh, P Chasela. University of Malawi, College of Medicine, P/Bag 360, Blantyre, Malawi

\subsection{6/sextrans-2015-052270.603}

Introduction Change of any drug regimen impacts on compliance. Malawi changed the first line ARV regimen from stavudine-based (stavudine + lamivudine + nevarapine) to tenofovirbased regimen (tenofovir + lamivudine + efavirenz) because the former was associated with adverse side-effects and poor compliance. This study aimed at assessing the impact of the new ARV regimen on compliance.

Methods Using cross-sectional study, 169 participants recruited from 6 ART clinics randomly selected were interviewed to assess views on compliance, side-effects and satisfaction to new regimen. Self-reported data on compliance was complemented with patients' records at the clinics.

Results Compliance was poor in first visits on tenofovir-based regimen but gradually improved. Side-effects like dizziness, drowsiness and nightmares were reported in 56.9\%, 24.9\%, $23.1 \%$ of participants respectively especially during the first weeks and these negatively affected compliance (odds ratio = 1.5). Mean individual adherence was $93.3 \%$ on tenofovir-based and $85.5 \%$ on stavudine-based regimen ( $\mathrm{p}$ Value of $<0.0001$ ).

Conclusion Tenofovir-based regimen has improved long term compliance and has the potential to eliminate suboptimal compliance rates to ARVs being a drug taken once daily. However 
patients still get transient side-effects especially at the beginning of taking this regimen due to efavirenz as such, regular monitoring and thorough counselling of all patients on the side effects of tenofovir-based regimen and transient nature of side effects is needed. A large scale study to be done to obtain data on longterm side-effects of tenofovir-based regimen most possibly renal impairment due to tenofovir or efavirenz-induced gynecomastia.

\section{P17.26 VIRAL LOADS AMONG HIV-INFECTED PERSONS DIAGNOSED WITH PRIMARY AND SECONDARY SYPHILIS IN FOUR US CITIES: NEW YORK CITY, PHILADELPHIA, PA, WASHINGTON, DC, AND PHOENIX, AZ}

\begin{abstract}
1,2,3 Melanie M Taylor*, 'Daniel R Newman, ${ }^{1,4}$ Julia A Schillinger, ${ }^{1,5}$ Felicia MT Lewis, ${ }^{1,6}$ Bruce Furness, ${ }^{4}$ Sarah Braunstein, ${ }^{4}$ Tom Mickey, ${ }^{2}$ Julia Skinner, ${ }^{5}$ Michael Eberhart, ${ }^{6}$ Jenevieve Opoku, ${ }^{1,4}$ Susan Blank, ${ }^{1}$ Thomas A Peterman. ${ }^{1}$ Centers for Disease Control and Prevention, Division of STD Prevention, Atlanta, GA, USA; ${ }^{2}$ Arizona Department of Health Services, STD Program, Phoenix, AZ, USA; ${ }^{3}$ Maricopa County Department of Public Health, STD Program, Phoenix, AZ, USA; ${ }^{4}$ New York City Department of Health and Mental Hygiene, Long Island City, NY, USA; ${ }^{5}$ Philadelphia Department of Public Health, Philadelphia, PA, USA; ${ }^{6}$ HIVIAIDS, Hepatitis, STD and TB Administration, District of Columbia Department of Health, Washington DC, USA
\end{abstract}

\subsection{6/sextrans-2015-052270.604}

The findings and conclusions in this report are those of the authors and do not necessarily represent views of the Centres for Disease Control and Prevention.

Background Incident syphilis among HIV-infected persons indicates ongoing behavioural risk for HIV transmission. Detectable viral loads among co-infected cases may amplify this risk.

Methods Primary and secondary (P\&S) cases reported during 2009-2010 from four US sites were cross-matched to local HIV surveillance registries to identify syphilis case-persons infected with HIV prior to or shortly after the syphilis diagnosis. We examined HIV viral load and CD4 results collected within 6 months before or after syphilis diagnosis for the co-infected cases identified. Independent correlates of detectable viral loads ( $\geq 200$ copies $/ \mathrm{mL}$ ) were determined.

Results We identified 1675 cases of incident primary or secondary syphilis among persons with HIV. Median age was 37 years, 99.5\% were male, 41.1\% were African American, 24.5\% Hispanic, and $79.9 \%$ of the HIV diagnoses were made at least one year prior to syphilis diagnosis. Among those co-infected, there were no viral load results reported for 188 (11.2\%); of the 1487 (88.8\%) with reported viral load results, 809 (54.4\%) had a detectable viral load (median 25,101 copies/mL, range 2063,590,000 copies $/ \mathrm{mL}$ ). Detectable viral loads were independently correlated with syphilis diagnosed at younger age, at an STD clinic, and closer in time to HIV diagnosis.

Conclusion More than half of syphilis case-persons identified with HIV had a detectable viral load collected within 6 months of the syphilis diagnosis. This suggests virologic as well as active behavioural risk for transmitting HIV.

\section{P17.27 DECENTRALISING HIV VIRAL LOAD TESTING TO A REGIONAL LABORATORY IN AGADIR, SOUTHERN MOROCCO}

${ }^{1}$ Houda Eloudyi ${ }^{*},{ }^{1}$ Sanae Lemrabet, ${ }^{2}$ Mohamed Aghrouch, ${ }^{2}$ Samira Kharbouch, ${ }^{1}$ Hicham Oumzil. ${ }^{1}$ National Referral Laboratory of HIV, National Institute of Health, Rabat, Morocco; '2aboratoryOf Biology, Hassan II Hospital, Agadir, Morocco

10.1136/sextrans-2015-052270.605
Despite the increase of HIV patient throughput in Morocco, follow up testing (HIV Viral load VL) is still centralised in the National referral laboratory (NRL) as the activity required trained staff and specialised infrastructure.

Patients were often lost due to great distances between testing centre and home as well as delays in returning results.

To follow the dynamic of decentralising HIV treatment and care in Morocco, the NRLH launched a process of strengthening regional laboratory capacities.

In this framework, we assessed factors associated with lab capacities to offer HIV viral load testing, and followed their performance after implementation.

On site visits were performed to the laboratory of the regional laboratory of the Hassan II hospital of Agadir in southern Morocco; this region that accounts the greatest number of HIV positive cases. Using Laboratory assessment tool, the laboratory capacities were evaluated, and gaps related to the facility layout, human resources, training, equipment and reagent were fixed thank to the Global Fund for HIV support.

A follow up the lab performance was set for a period of two months, by retesting all the samples $(n=194)$ at the NRL. Data were analysed using MedCalc software to calculate the Spearman's coefficient of rank correlation (rho).

VL results were ranged from 150 to 221242 copies $/ \mathrm{ml}$.

A perfect match of VL results between the measurements at the NRL and at the Agadir regional lab was observed. The observed Spearman correlation index was of 0.98. P $<0,0001$ (95\% CI: 0,973 to 0,990 ).

In the light of these results, HIV VL testing was moved to the periphery and closer to the site of therapeutic management structures. Patients could get their results conveniently and quickly. This experience is worth to reproduce in other regions of the kingdom were HIV prevalence is steadily increasing.

\section{P17.28 ASSESSMENT OF HIV-1 PRIMARY DRUG RESISTANCE MUTATIONS IN ANTIRETROVIRAL THERAPY-NAIVE CASES IN MOROCCO}

${ }^{1} \mathrm{H}$ Eloudyi*, 'S Lemrabet, ${ }^{2} \mathrm{M}$ Malmoussi, ${ }^{2} \mathrm{Z}$ Ouagari, ${ }^{1} \mathrm{E}$ Elharti, ${ }^{1} \mathrm{M}$ Akrim, ${ }^{1} \mathrm{H}$ Oumzil. ${ }^{1}$ Laboratoire National de Référence Pour Le VIH. Institut National d'Hygiène, Rabat; ${ }^{2}$ Centre Référent VIH, Hôpital Hassan II, Agadir

\subsection{6/sextrans-2015-052270.606}

Antiretroviral drug resistance is a major challenge for management and control of HIV-1 infection worldwide and particularly in resource limited countries.

Although combined antiretroviral drug therapy has greatly improved the life-span and the life quality of the patients, HIV-1 drug resistance poses a major obstacle for treatment outcome.

This study aims to determine the prevalence of primary resistance in a group of newly diagnosed patients naïve to treatment, in the region of Souss Massa Draa in sounthern Morocco.

A total of 47 antiretroviral treatment (ART) naïve patients were included. Virological status was determined by Real time PCR (Abbott, USA).

Primary drug resistance mutations were identified according to the Stanford HIV database and the algorithm of the National Agency for AIDS Research (ANRS). The clinical staging of patients was made upon the International Classification of the Centres for Disease Control (CDC).

Ninetheen $19 \%$ of recruits were diagnosed in stage A, $11 \%$ in stage $\mathrm{B}$, and the large proportion of patients in stage C $38 \%$, while $32 \%$ of which the stadium has not been determined. 